
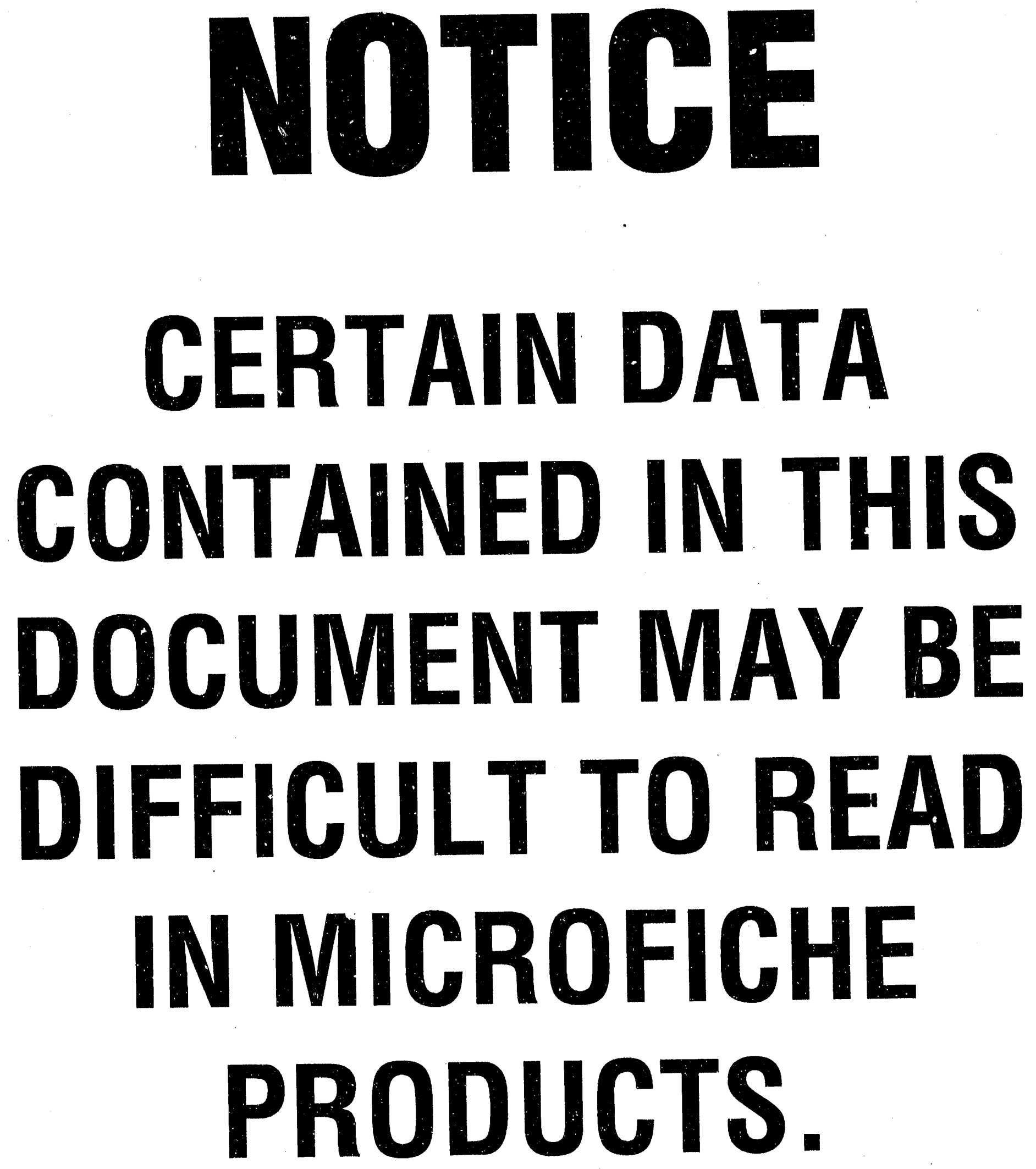
LA-UR $-90-4168$

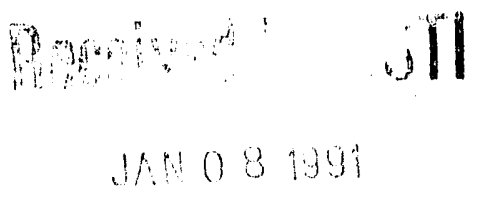

$\because$

DE9 1005895

TITLE. USE OF A GENETIC ALGORITHM TO ANALYZE ROBUST STABILITY PROBLEMS

AUTHOR(S): T. M. Murdock

W. E. Schinitendorf

S. Forrest

SUBMITTED TO: for the Proceedings of the American Automatic Control Conference, to be held June 91, in Boston, MA.

\section{DISCLAIMER}

This report was prepared as an account of work sponsored by an agency of the United States Government. Neither the United States Gnvernment nor any agency thereof, nor any of their employees, makes any warranty, ; press or implird, or assumes any legal liability or responsibility for the accuracy, completeness, or usefulness of any information, apparatus, product, or process tisclosed, or represents that its use would not infringe privately owned rights. Reference herein to any specific commercial product, process, or service by trade name, trademark, manufacturer, or otherwise does not necessarily constitute or imply its endorsement, recommendation, or favoring by the United States Government or any agency thereof. The vie:vs and opinions of authors expressed herein do not necessarily state or reflect those of the Lrited States Government or any agency thureor.

\section{MASTER}

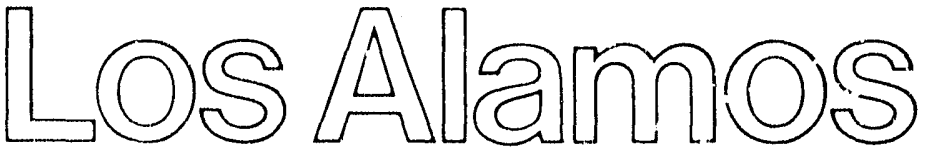




\title{
Use of a Genetic Algorithm to Analyze Robust Stability Problems
}

\author{
T. M. Murdock, W. E. Schmitendorf \\ University of California-Irvine \\ Irvine, CA 92717 \\ and \\ S. Forrest \\ University of New Mexico \\ Albuquerque, NM 87131
}

\begin{abstract}
This note presents a genetic algorithm technique for testing the stability of a characteristic polynomial whose coefficients are functions of unknown but bounded parameters. This technique is fast and can handle a large number of parametric uncertainties. We also use this method to determine robust stability margins for uncertain polynomials. Several benchmark examples are included to illustrate the two uses of the algorithm.
\end{abstract}

\section{Introduction}

Stability of polymomials plays an important part in the analysis and design of control systems. A standard approach to robustness analysis of linear dynamic systems is to examine the characteristic polynomial in the presence of parametric uncertainties. The characterstic polynomial is of the form

$$
P(s, \mathrm{q})=\sum_{m=0}^{\mathrm{n}} a_{m}(\mathrm{q}) s^{m}
$$

where $q=\left[q_{1}, q_{2}, \ldots, q_{1}\right]^{T}$ is a vector of uncertain parameters which are contained in the compact set $Q=\left\{q: q_{i} \in\left[q_{i}^{-} ; q_{i}^{+}\right] ; i=1,2, . l\right\}$. The coefficient functions $a_{m}(q)$ can vary linearly, multilinearly or polynomically with $q$. Although it exceeds the scope of this paper, we believe the algorithm can be applied to almost an functions $a_{i}(q)$.

For a particular value of $q$, the polynomal is said to be stable if all of its roots lie in the strict left half complex plane. Similarly, in order to be robustly stable, the roots of the polyomial must be contained in the strict left half plane: $\forall \mathrm{q} \in \mathrm{Q}$. This means that the maximum real root component of the rests (hereafter refered to as the maximum root) of the characteristic polynomial must also lie in the left half plane $\forall q \in Q$. In this paper an optimization scineme known as a genetic algorithm (GA) (1) is applied to this problem of finding the maximum root of $P(s, q)=0 \forall q \in \mathrm{Q}$. If this maximm root is positive then the polynomial is unstable, othewise we conclude the polynomial is stable. The algorithm allows 
for interval, linear affine, multilinear, and polynomic coefficient functions in $q$. The algorithm will also determine the vector $\mathrm{q}_{\max }$, which yields the maximum root. Polynomials of the form 1 also arise when analyzing the stability of a system in state space form. If $\dot{x}(t)=A(\mathrm{q}) x(t)$, then the characteristic polynomial $P(s, \mathrm{q})=\operatorname{det}[s I-A(\mathrm{q})]=\sum_{m=0}^{n} a_{m}(\mathrm{q}) s^{m}$ anci this polynomial is used in checking stability.

In addition to the application of testing stability for a specified $\mathbf{Q}$, we present an extension of this technique which is applied to the problem of measuring the robust stability margin of characteristic polynomials with uncertain coefficient functions. The robust stability margin is denoted in this paper by $\bar{r}$, where $\bar{r}$ is the largest value of $r$ such that $P(s, \mathrm{q})$ is stable for all $\left|q_{i}\right| \leq \bar{r}$. It is also commonly thought of as the largest hypercube in parameter space which yields robust stability. The most important aspects of the genetic algorithm are that it presents a numerically fast method of testing robust stability for characteristic polynomials with any type of coefficient function that varies polynomially in $q$, and it can treat a large number of uncertain parameters.

\section{Background}

Techniques based on necessary and sufficient conditions exist for stability determination of characteristic polynomials whose coefficient functions are interval polynomials or linear affine functio's in $q$. In the case of interval polynomic coefficients, Kharitonov's theorem is necessary and sufficient [2]. It allows us to determine the stability of the entire Qbex by checking the stability of only four polynomials. The Edge Theorem is a necessary and sufficient test for problems with $a_{i}(q)$ linear affine functions (as well as the interval polynomial casc). The Edge Theorem states that we can test stability of the entire Q-box simply by testing the stability of the exposed edges of the Q-box [3].

Tests for cases where the coefficient functions are multinear or polynomic in $q$ are also available. One such test is the concept of zaro inclusion (4). This occurs when the value set of a polynomial, which is obtained by mapping the Q-box into the complex plane for a given complex point $\hat{s}$, contains the origin $0+0 i$. The zero inclusion test itself is necessary and sufficient for all classes of polynomials mentioned above; however the implementations of a zcro inclusion test, such as the $\mathrm{H}$-function test of Barmish [5], are not. Another test which is recessary and sufficient for any form of coefficient function is the robust root locus. The robust root locus is a plot of the root space of the characteristic polynomial $\forall q \in Q$. It can be generated using a zero inclusion test [G], or by brute force gridding of the $Q$-box. The zero inclusion and robust root locus tests are necessary and sufficient for coefficient functions which are interval, lincar affine, multilinear or polynomic in $q$. The value set, however, may be difficult to detcrmine (i.c. may require gridding). Use of the zero inclusion test simplifies in the case of interval and linear affine polynomials, since the value set is equivalent to the convex hull of the vertices of the Q-box mapped to the complex plane. Thus, the problem of checking for zero inchusion in these cases reduces to checking whether zero is included in the convex hull (a much simpler check). For the multilinear case, the value set is contained within the convex hull and thus may yield conservative results (i.e. may conclude matiale when stable). For the polynomic ase, these simplifications do not, hold and use of the eomex lubll way be completely in error (i.e. may conchade stable when unstial)le). 
All of the techniques previously mentioned work well for interval and linear affine functions in $q$, but they are at best conservative tests for multilinear functions and can completely fail where the coefficient functions are polynomic. Domain splitting techniques [7] can be used to reduce the conservatism for multilinear cases, and can help increase accuracy for polynomic cases as well. Additional stability tests which eliminate conservatism for polynomials with multilinear and polynomic coefficient functions are also available $[8,9]$ The computer-aided method presented in $[8]$ is based on the parameter space approach developed in [10]. It is promising in that it can handle polynomic coefficient functions although it still may suffer from extensive computation time if a large number of uncertain parameters are present or dense gridding is required. This method is exten ively evaluated in $[10,11]$. Another method of computer-aided stabilty analysis uses a value set approach which requires mapping the Q-box into the complex plane for selected frequencies and checking for zero inclusion. This method is attractive for polynomials with polynomic coefficient functions, but it will work only if the polynomial in cuestion admits to a tree structured decomposition [9]. Note that it is not always possible to determine a tree structured decomposition.

In general, for polynomials with large numbers of parametric uncertainties [12], most stability tests become computationally intractable. Large numbers of perturbations can influcnce the effectiveness of a test by creating a "combinatoric explosion" in the number of tests to be performed. Barmish points out in (13) that when using the edge test on linear. affine polynomials, a combinatoric explosion in the number of exposed edges as a functjon of the number of perturbation parameters $l$ can occur. That is, for an $l$-dimensional Qbox, $l 2^{l-1}$ edges must be checked for stability. Combinatoric explosions in calculations are more evident when using the brute force approach of gridding the Q-box and finding the roots at every point. Calculation times for using this technique can grow large even for a small number of parameters when a dense grid is being used. This type of test is used for comparison in cases where no necessary and sufficient condition test is appropriate. Here we show that a GA based stability test will solve problems for which only conservative results could be obtained using existing tests. In ardition, this technique is faster then existing techniques. It avoids combinatoric explosions in calculations caused by the presence of a large number of uncertainties. Lastly, it solves benchmark problems which challenge the most current analytical stability tests.

The problem of parametric perturbations and their effect on stability margin has also been widely studied. For the class of interval matrices, sufficient conditions for stability are given in $[14,15]$, where Lyapunov approaches are used, and also in [16] where the Gershgorin theorem is applied. Lyapunov based approaches can also be used on linearly structured matrix perturbations to derive stability spheres in paraneter space [17]. When the macertain parameters occur polynomially in matrix mintes, references [18] and [19] offer a complete solution to checking eigenvalue location in the presence of one or two uncertain parameters. However, [18] states that, when the mumber of mocertain paraneters exceeds two, this technique becomes cumbersome from is iomputational burden point of viow. More recently, an algorithm for detemining stability perturbation boumds for systems with uncertainties entering the state equation matrix nonlinean!y has becn presented [20)]. This algorithm transforms the stability margin nonlineat froblem inter a modified signomial programming problem [21] which offers consistent convergence speed inprovements and can handle larger numbers of parametric uncertaintics. Heweres, it is still stew companed tor the GA and, we believe, more complicated to use. 
Using the GA, we show that the robust stability margin can be determined for any of the previously mentioned classes of coefficient functions. The GA technique is also much faster and more generalized than current methods. This implies that stability margins can be calculated for characteristic polynomials with large numbers of uncertain parameters as well. It also seems reasonable that this technique can be extended to determine a robust stability margin based on a specified region, $\Gamma$, in the complex plane ( $\Gamma$-stability).

\section{Genetic Algorithm}

Genetic algorithms were initially developed by Holland [22] and comprehensive descriptions of GAs are found in $[1,23]$. A GA is a search algorithm based on the principles of Darwinian evolution. GAs work by selecting a random sample of string structures ( $q$ ), evaluating their worth (determined by the user in a fitness function), and stochastically selecting a. new sample based on the information provided by the previous iteration. The algorithm combines the principle of natural selection (by eliminating the least fit structures fiom the sample) with "genetic" operators such as mutation and cross-over which choose the new sample points at each iteration. We used the GENESIS implementation [24,25] of the GA for the reported work.

The GA is used in stability determination as an objective function minimization routine. That is, the algorithm determines the structure (vector $\mathrm{q}$ ) that corresponds to the minimum fitness evaluation. The user supplies the fitness function as well as the bounds and precision on the domain of structures (e.g., uncertain parameters $q_{i}$ ). The uncertain parameters are converted to bit strings (structures) for processing by the GA. The conversion process involves several steps: (1) mapping the bounds and precision of each parameter into discrete intervals, (2) producing the binary encoding of the interval corresponding to each $q$ in the vector, (3) converting each binary encoding to its Grey code equivalent, and (4) concatenating the resulting Grey codes to produce one bit string. The precision of the uncertain parameters is controlled by the length of the bit string (i.c., the longer the bit string, the more precise the parameters are). In our application, the fitness function consists of the uncertain coefficients (in $q$ ) of the characteristic polynomial, at root finding algorithm, and a root sorting scheme to determine the maximum root (root furthest to the right). The structures are sent to the fitness function, where the maximum root is detcrmined and retained for the set of roots corresponding to each q. The maximization problem is converted to a minimization problem by multiplying the maximum root by negative unity. Thus, by finding the vector $q_{\max }$ that corresponds to the best (lowest) fitness, we are actulially finding the vector that produces the maximum root.

The Q-box is specified by the bounds on the individual components of the a vector. Several random points in the Q-box are chosen as an initial population of structures, and the maximum root is determined for each vector q. The position of the maximum root. determines the fitness of the vector $q$. The GA scarches the $Q$ box for the frobal optimum by increasing the chance of reproducing fit, structures and reducing the chance of reprociucing unfit structures. The genetic operators "cross-over" and "mutation" are used during reproduction of each new generation. The cross-over operator exchanges characteris. tics (contignous bits) between two fit structures in the sane manner that cosss-over anong chromosomes exchanges genetic information during meiosis. The ress-over operator allows 
the search to avoid converging on local optima by making structured long jumps in the search space. Mutation, on the other hand, sclects bits randomly and flips their values. This serves as a randomization procedure and prevents the search from becoming frczen at certain values (i.e., if all the structures in a generation have bit position 6 set to 0 , then without mutation, bit position 6 would be set to 0 in all successive generations). The rates at which cross-over and mutation are applied are user-specified, although the mutation rate is typically much lower than the cross-over ratc. The GA is a surprisingly fast and accurate technique for searching the Q-box to obtain the vector $q$ which yiclds the maximum root.

Since the $G A$ is a heuristic sampling algorithm designed to escape local optima, it does not suffer from the problems associated with techniques based on derivatives or line searches. By combining the cross-over and mutation operators, the GA has a much better chance of eventually finding a global optimum. For example, assume the GA is searching a twodimensional Q-box. If it begins converging on a local optimum (by reproducing the most fit individuals), the randomess inherent in the cross-over and nutation operators will cause the GA to randomly search elsewhere in the Q-box to avoid overlooking other optima. While this gives us a high degree of confidence that the global optima is eventually found, it should be noted that there is no guarante that the GA will not become trapped in a local optimum.

\section{$4 \quad$ Stability Analysis}

Robust stability analysis of polynomials with interval and linear affine coefficient functions using the $G A$ was verified against known results (using Kharitonov's theorem and the Edge test). The GA was able to correctly determine whether or not a polynomial was stable. The results for multilinear and polynomic coefficient functions are much more interesting because no analytic results are available for checking stability. In this section, we present our results for several such examples.

\section{Example 1: A Multilinear Polynomial}

A classic example of a multilinear polynomial which is difficult to solve using known methods is that of the unstable enclave $(10,11]$. The challenge of this example is that an unstable ball is contained in the Q-box, while the edges of the Q-box remain stable. The enclave can be made arbitrarily small and avoids detection by edge tests and brute fores: grictding.

Consider the thind order characteristic polynomial with $l$ parametric uncertainties $y_{i}$

$$
P(s, q)=a_{0}(q)+a_{1}(q) s+a_{2} q s^{2}+s^{3}
$$

where

$$
\begin{aligned}
& a_{11}\left(q_{1}\right)=1(1-1)+r^{2}+2(l+1) \sum_{i=1}^{1} q_{i}+2 \sum_{i<j}^{1, \ldots, l} q_{i} q_{j} \\
& a_{1}\left(q_{1}\right)=1+\sum_{i=1}^{1} q_{i} \\
& a_{2}\left(q_{1}\right)=a_{1}(q)
\end{aligned}
$$


The Routh criteria of $a_{1} a_{2}-a_{0}>0$ yields the stability constraint $\sum_{i=1}^{l}\left(q_{i}-1\right)^{2}>r^{2}$, thus $r$ is the radius of a ball of unstable points in $\mathbf{Q}$ that is centered at $\mathbf{q}=[1,1, \ldots 1]^{T}$. Note that if $r=0$, then a single unstable point exists at $\mathrm{q}=[1,1, \ldots 1]^{T}$, and if $r^{2}$ is replaced by any negative number the polynomial becomes stable $\forall q \in Q$. This example is interesting because it allows us to vary the radius of the unstable ball $r$ as well as the number of uncertain parameters $l$. The accuracy of the GA can be evaluated by shrinking the enclave to be arbitrarily small. In addition, its ability to handle a large number of parameters can be tested by increasing $l$.

The accuracy of the uncertain parameters in this example was set to twelve bits of resolution per $q_{i}$ which divides each user-specified parameter range into $2^{12}$ (4096) points. Results of applying the GA to this example show that, for two parameters, an enclave of radius $r=1 \times 10^{-5}$ can be detected. When the radius of the enclave was set to zero, the GA verified that the system was unstable and the vector corresponding to the maximum root was determined to be $\mathrm{q} \approx[1,1]^{T}$. Conversely, when the term $r^{2}$ was replaced with $-1 \times 10^{-4}$, the GA verified that the polynomial was stable. Figure 1 shows the evolution of the best fitness (maximum root) and the average fitness (average of the maximum roots of the entire population) over 300 generations for the two parameter case with the enclave set to zero.

Next, the problem was considered with a large number of parameters. For 10 uncert sin parameters, an enclave of radius $r=1 \times 10^{-2}$ was detected (Figure 2). More importantly, using the GA with the same enclave radius, we were able to cietect instability for up to 50 uncertain parameters! This proves that the that the GA can handle a larger number of parametric uncertainties than any other stability test to date.

The size of the minimum radius that was detected for the two parameter case does not represent limitations of the GA. Instead, the minimum radius is used to determine a grid size necessary to detect the enclave using a brute force gridding technique. The GA is compared to gridding because the domain of the fitness function is effectively gridded by setting the length of the bit strings representing the $q_{i}$ 's. It accomplishes its savings over gidding by sampling poirts in a more intelligent fashion, thus adding some of the innovative flair of a human scarch. By comparing, the GA with brute force gridding, we can illustrate that the GA avoids a combinatoric explosion in calculations. In the two parancter case with the cnclave radius $r=1 \times 10^{-5}$, grideling the intervals $q_{1}=[0.0 ; 2.2], q_{2}=[0.0 ; 3.0]$ would require a grid of $6.6 \times 10^{10}$ gridpoints. On an Apollo 3500 , assuming $3 \mathrm{~ms}$ for coefficient and root calculations, the computational effert. would take two years. The calculation tine necied for the GA in the two paraneter cisce was muder two minutes on the Apollo 3500. In the case of ten paranetric uncertainties, the (i A recuired approximately six minutes to run. For fifty parancters, the mustable coldave was detected in thirty minutes on the sime Apollo 3500 machine. Granted that other metheds previously mentioned are superior to brute force gridding in terms of calculation time, lacy may still yicld conservative resultsis for a polynomial with multilincir coefticient functions in q. Certainly, none of the nethods mentioned carlier are capable of handling a multilinear problem with 50 uncertain parane. ters. The GA, on the other hand, is heth fast (5) uncertain paraneters in thirty minutes) and applicable to a mose gerera! elassi of prestilents.

Example 2: A Polymomir Polymunial

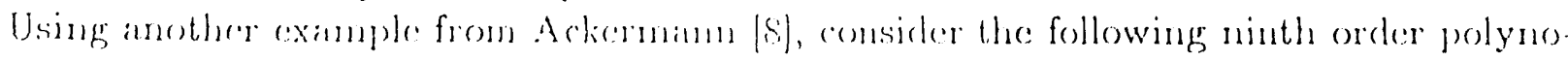


mial with the coefficient functions

$$
\begin{aligned}
& a_{9}(\mathrm{q})=1+0.02\left(q_{1}^{2} q_{2} q_{3}^{2} q_{4}^{2}\right) \\
& a_{8}(\mathrm{q})=51\left(1+0.04\left(q_{1}^{2} q_{2} q_{3} q_{4}\right)\right) \\
& a_{7}(\mathrm{q})=1156\left(1+0.05\left(q_{1}^{2}+q_{2}+q_{3}\right) q_{4}\right) \\
& a_{6}(\mathrm{q})=15260\left(1+0.05\left(q_{1}^{2}+q_{2}+q_{4}\right) q_{3}\right) \\
& a_{5}(\mathrm{q})=128963\left(1+0.05\left(q_{1}^{2} q_{4}+q_{2} q_{3}\right)\right) \\
& a_{4}(\mathrm{q})=721505\left(1+0.05\left(q_{1}^{2} q_{3}+q_{2} q_{4}\right)\right) \\
& a_{3}(\mathrm{q})=2664608\left(1+0.05\left(q_{1}+q_{2}+q_{3}\right) q_{4}\right) \\
& a_{2}(\mathrm{q})=6253816\left(1+0.05\left(q_{1}+q_{2}+q_{4}\right) q_{3}\right) \\
& a_{1}(\mathrm{q})=8445840\left(1+0.1\left(q_{1} q_{4}+q_{2} q_{3}\right)\right) \\
& a_{0}(\mathrm{q})=4934800\left(1+0.1\left(q_{1}+q_{2}+q_{3}+q_{4}\right)\right)
\end{aligned}
$$

$\mathrm{n}[8]$, the parameter intervals for $q_{3}$ and $q_{4}$ are fixed at $[-1.0 ; 1.0]$ and it is shown that the polynomial is stable for $q_{1} \in[-0.8 ; 1.8], q_{2} \in[-0.8 ; 1.2]$. Using these intervals and the GA algorithm, the maximum real component of the root was found to be $-1.239 \times 10^{-1}$, indicating stability (Figure 3), and occurred for $\mathrm{q}=[-0.79492,-0.79902,-0.99707,0.99601]^{T}$. By changing the $q_{1}$ interval to $q_{1} \in[-1.1 ; 1.8]$, the polynomial became unstable (Figure 4 ) and the maximum real root component, $2.675 \times 10^{-2}$ occurred at $q=[-1.1,-0.8,-1.0,1.0]^{T}$. Thus, the GA can effectively analyze this polynomial with polynomic coefficients.

The comp atation time required for this example was approximately fifteen minutes on the Apollo 3500 for both the stable and unstable cases. The computer-aided method presented in $[8]$ takes two hours to rin on the Apollo 3500 for the same example, but also provides more information for stability bounds when only two of the parameters are allowed to vary, but the other two are fixed.

While the GA provides less information in the two paraneter variation case, it does allow us to vary all four parameters simultaneously instead of fixing $q_{3}$ and $q_{4}$ and examining the remaining parameters. This gives us the added benefit of being able to find the largest hypercube for which the above polynomial is stable $\left(r^{*}=0.955\right)$. (The computation of rolust stability margin is discussed more fully in the next section.) This versatility can ultimately give us more information about the liniting stability values for sach individual pirimeter.

\section{Robust Stability Margin}

Fron the previous examples, we sec that this technique can be extended to determine the robust stability margin for a system (i.e. the largest hypercube in paraneter spare for which 1.he characteristic polynomial is stable). Using the GA, the stability margin fon a given chanteristic polynomial can be cetablished through simple iteration. The hypercube is

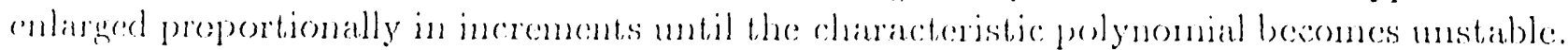
The hepercube is then reduced in smaller increments mitil the polynomial becomes stable again. The accuracy of the stability margin is controlles by the number of iterations the user maties. Calcuation times vary, depending on the order of the polynomial more than the munber of parancters since the calculation of the roots of a high order polynonial can 
be time consuming. Two examples taken from [7,20,27], illustrate the effectiveness of using the liA to conpute robust stability narign.

Example 3: A Multilinear Cóefficient Polynomial

Consider the following coefficient functions for a fourth order monic polynomial.

$$
\begin{aligned}
& a_{0}(\mathrm{p})=2 p_{1} \\
& a_{1}(\mathrm{p})=10 p_{2} p_{3}+p_{1} \\
& a_{2}(\mathrm{p})=p_{2} p_{3}+10 p_{2}+10 p_{3} \\
& a_{3}(\mathrm{p})=10+p_{2}+p_{3}
\end{aligned}
$$

with

$$
\begin{aligned}
& p_{1}=800+800 q_{1} \\
& p_{2}=4+2 q_{2} \\
& p_{3}=6+3 q_{3} .
\end{aligned}
$$

This example arose in the detemination of the $l_{\infty}$ stability margin for a closed loop system [27]. We would like to determine the largest $\bar{r}$ where $\left|q_{i}\right| \leq \bar{r}^{*} ; i=1,2, \ldots l$ for which the fourth order polynomial. $P(s, \mathrm{p})$ is stable. By iteration, we find that the polynomial is unstable for $\ddot{r}=0.341 \mathrm{~s}$ and stable for $\vec{r}=0.3416$. One could iterate even further for a more exact measure of stability margin. This compares well to the results of [20], where $i$ was found to be 0.3417 . The computational time for this example cannot be compared to that of the algorithm presented in [20], as the GA was run on a different system.

Example 4: A Polynomic Coefficient Polynomial

In [7], a fourth order monic polynomial is studied with the coefficient functions

$$
\begin{aligned}
& a_{0}(p)=p_{3}^{3} \\
& a_{1}(p)=p_{1} p_{2}^{3} p_{3}^{2} \\
& a_{2}(p)=p_{1}^{2} p_{2}^{2} p_{3} \\
& a_{3}(p)=p_{1}^{3} p_{2}
\end{aligned}
$$

where

$$
\begin{aligned}
& p_{1}=1.4+0.25 q_{1} \\
& p_{2}=1.5+0.2 q_{2} \\
& p_{3}=0.8+0.2 q_{3} .
\end{aligned}
$$

Using the CA and iterating to find the largest hypercube yields a stability margin of $\because=1.09$. Onee again, the computing time lepends on the accuracy desired by the user and is difficult te compare with the algerithm in $(20)$; however, we believe the $G A$ is simpler conceptually and can accommodate langer mumbers of paranctric uncertanties. 


\section{Conclusions}

In this paper we have addressed the problem of robust stability analysis of linear dynamic systems. We used a genetic algorithm to find the maximum root of the system's characteristic polynomial in the presence of uncertain parameters. We believe that it is supcrior to present stability tests for the following reasons: (1) The coefficient functions may be any of the four classes of polynomial functions (interval, linear affine, multilinear, polynomic) mentioned previously. (2) The coefficient functions can contain a large number of parancters without producing a combinatoric explosion in calculations. (3) The GA is an intelligent search which produces results faster than existing techniques.

This algorithm can be used to determine robust stability for polynomials which previously presented difficulties due to a combinatoric explosion in the number of calculations involved, or to the lack of an appropriate necessary and sufficient condition test. In addition, the GA minimization scheme was extended to determine the robust stability margin of characteristic polynomials with polynomial coefficient functions in $q$. It proved to function as well as complicated numerical techniques $[7,20,27]$, which are not nearly as versatile in the types of problems they can handle. The GA can be extended to $\Gamma$-stability problems and shows promise of being applicable to any controls problem which lends itself to a minimization scheme. While we have not shown that the GA is the best algorithm, our success on many examples shows that it merits consideration in analyzing robust stability problems.

\section{Acknowledgement}

This research was supported by Los Alamos National Laboratory under the INCOIR Program. The authors thank Dr. David K. Camploll for his support under this program.

\section{References}

[1] D. E. Goldberg, Genetic Algorithms in S'atch, Optimization, and Machine Learning, Addison-Wesley, Reading, Mass., 1989.

[2] V. L. Kharitonov, "Asymptotic Stability of an Equilibrium Position of a Fanily of Systems of Linear Differential Equations," Differential'nye Uraveniya, Vol. 14, No. 11, pp.1483-1485, 1978.

[3] A. C. Bartlett, C. V. Holiot, and L. Lin, "Resot Locations of an Entire Polytope of Polynomials: It Suffices to Check the Folges," Mathematics of Control, Signals, and Systems, Vol. 1, p1). 61-71, 1987.

(4) L. A. Zadeh and C. A. Desoer, Linear System. Theory, McGraw Hill, 1963, Section 9.17.

[5] B. R. Barmish, "Now Tools for Robustnusis Analysis," Proc. IEEE Conf. Decision and Control, Austin, Texas, pp. 1- 6, Dece 1988.

[6] B. R. Bamish and R. Tempo, "The Robust Root Locus," Proc. IEEE Conf. Decision and Control, Anstin, pp.1380 1391, Dece 1988. 
17] A. Sideras and R. S. Pena, "Fast Computation of the Multivariable Stability Margin for Real Interrelated Uncertain Parameters," IEEE Trans. Auto. Contr., Vol. 34, pp. 1272-1276, 1989.

[8] J. E. Ackermann and W. Sienel, "Computer-aided Robustness Analysis for Characteristic Polynomials with Polynomial Parancter Dependence," Proc. IEEE Conf. Decision and Control, Tampa, Fla., pp.1934-1935, Dec. 1989.

[9] B. R. Bannish, J. Ackermann, and H. Z. Hu, "The Tree-Structured Decomposition, A New Approach to Robust Stability Analysis," DLR-Report 515-89-10, 1989.

[10] J. E. Ackermann, D. Kaesbauer and R. Muench, "Robust I-stability Analysis in a Plant Parameter Space," DLR-Report 515-89-12, 1989, to appear in Automatica.

[11] J. E. Ackermann, H. Z. Hu, and D. Kaesbaner, "Robustness Analysis: A Case Study," Proc. IERE Conf. Decision and Control, Austin, Texas, pp. 123-128, Dec. 1989.

[12] J. E. Ackermann and W. Sienel, "What is a "Large" Number of Parameters in Robust Systems?," Proc. IEEE Conf. Decision and Control, Honslulu, Hawaii, Dec. 1990.

[13] B. R. Bam mish, "A Generalization of Kharitonov's Four Polynomial Concept for Robust Stability Problems with Linearly Dependent Coefficient Perturbations," Proc. Amer. Contr. Conf., Atlanta, Ga.,1988.

[14] R. K. Yedavalli, "Perturbation Bounds for Robust Stability in Linear State Space Modclis," Int. J. Contr., Vol. 42, pp.1507-1517, 1985.

[15] K. Zhou and P. Khargonekar, "Stability Robustness Bounds for Linear State- Space Models with Structured Uncertainty," IEEE Trans. Automat. Contr., Vol. AC-32, pp. 621-623, 1987 .

(16) M. B. Argoun, "On Sufficient Conditions for the Stability of Interval Matrices," Int. J. Contr., Vol. 44, pp. 1245-1250, 1986.

[1.7] L. H. Keel, S. P. Bhattacharyya, and J. W. Howze, "Robust Control with Structured Perturbations," IEEE Truns. Automat. Contr., Vol. 33, pp. 68-78, Jan. 1988.

[18] A. Tesi and A. Vincino, "Robust Stability of State-Space Models with Structured Uncertainties," IEEE Trans. Automat. Cuntr., Vol. 35, Feb. 1990.

[19] R. Genesio and A. Tesi, "Results on the Stability Robustness of Systems with State Space Perturbations," Syst. Contr. Lett., Vol. 11, pp. 39 47, 1988.

[20] A. Vicino, A. Tesi, and M. Milanese, "Computation of Nonconservative Stability Perturbation Bomds for Systems with Nonlincarly Corrclated Uncertainties," IEEE' 'Trans.s. Automat. Conti, Vol. 35, No. 7, p1). 835 841, July 1990.

[21] J.E. Falk, "Global Solutions of Signomial Programs," George Washington Univ., Washington, DC, Tech. Reps. T-274, 1973. 
[22] J. H. Holland, Adaptaiion in Natural and Artificial Systems, The University of Michigan Press, Ann Arbor, MI, 1975.

[23] K. Kirshnakumar and D. E. Goldberg, "Genetic Algorithms in Control Systerı Optimization," AIAA Guidance, Navigation and Contral Conf., pp.1568-1577, Portland, OR, Aug.1990.

[24] J. J. Grefenstette, "GENESIS: A system for using genetic search procedures," Proc. of the 1984 Conf. on Intelligent Systems and Machines, pp. 161-165.

[25] J. J. Grefenstette, "A user's guide to GENESIS," (Technical Report No. C.S-84-11), Nashville; Vanderbilt University, Depariment of Computer Science.

[26] J. E. Ackermann, "Does it Suffice to Check a Subset of Multilinear Parameters in Robustness Analysis?," Department of Electrical and Computer Engineering, University of California, Irvine, CA., 1989.

[27] R. R. E. de Gaston and M. G. Safonov, "Exact Calculation of the Multiloop Stability Margin," IEEE Truns. A utomat. Contr., vol.33, pjs. 156-171, 1988. 


\section{List of Figures}

- Figure 1. Fitness Evolution for 2 Parameters, Enclave Radius =0

- Figure 2. Fitness Evolution for 10 Prameters, Enclave Radius $=0.01$

- Figure 3. Fitness Evolution for Example 2, Stable Case

- Figure 4. Fitness Evolution for Exarnple 2, Unstable Case 


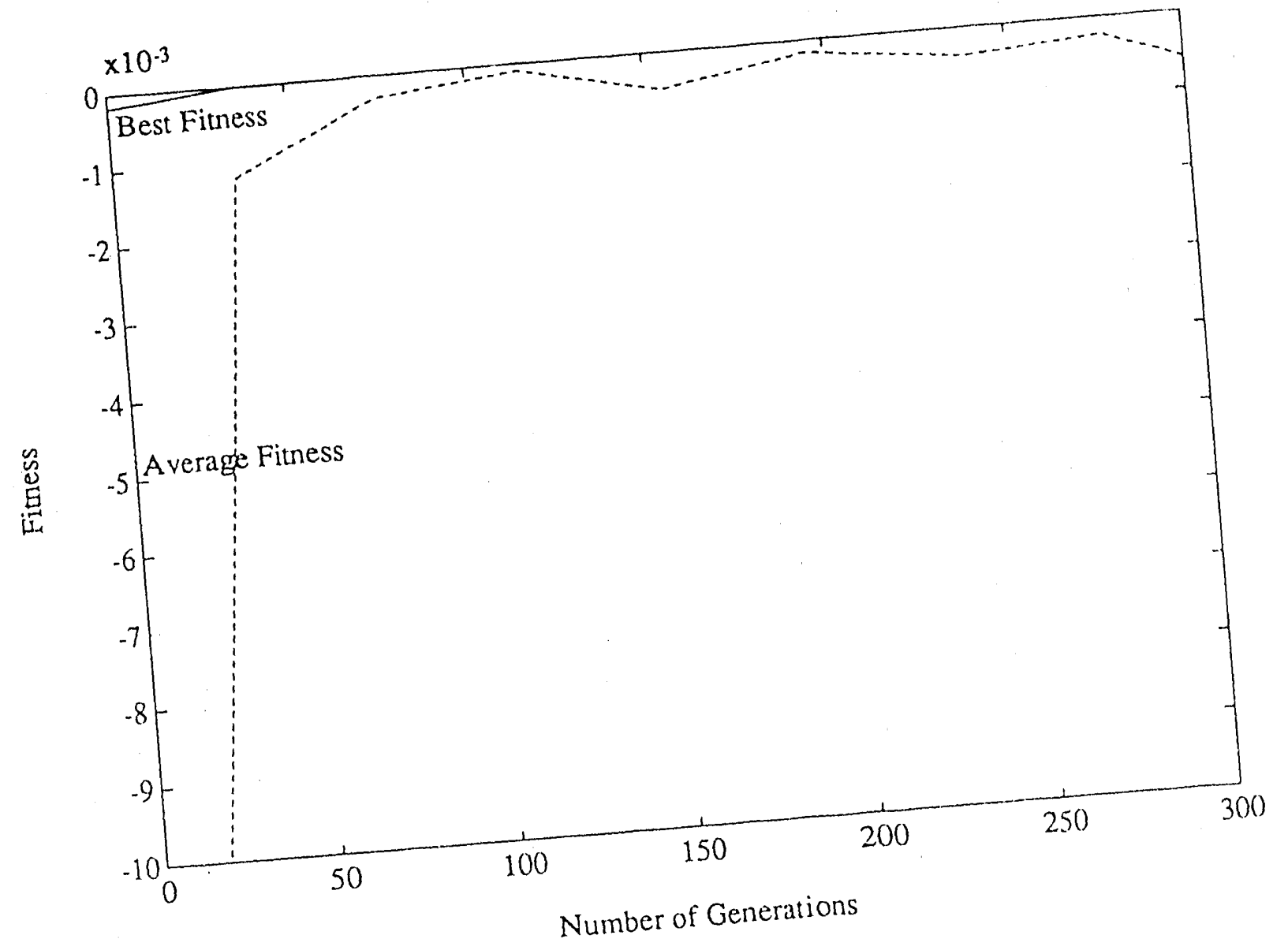




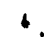

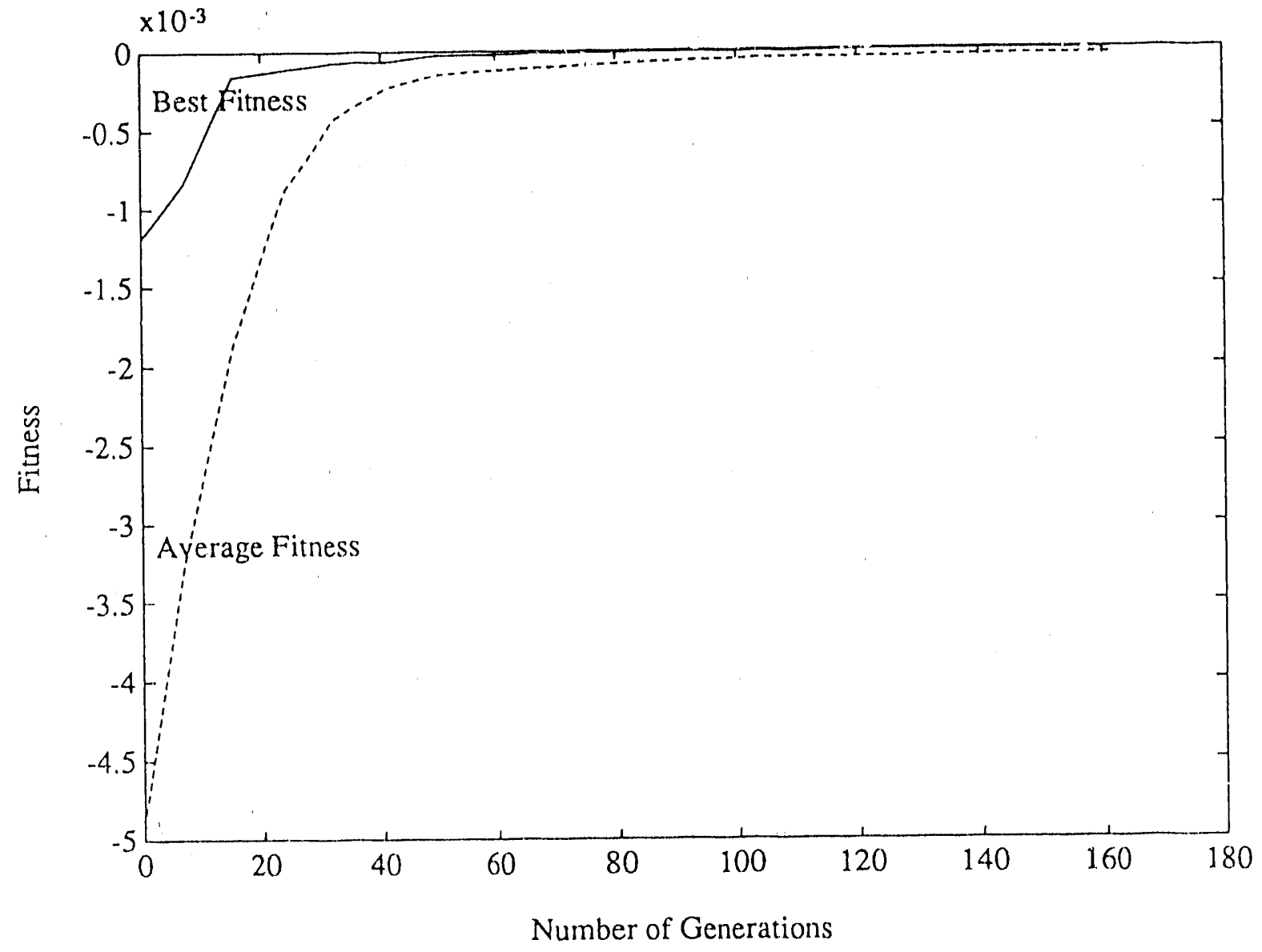




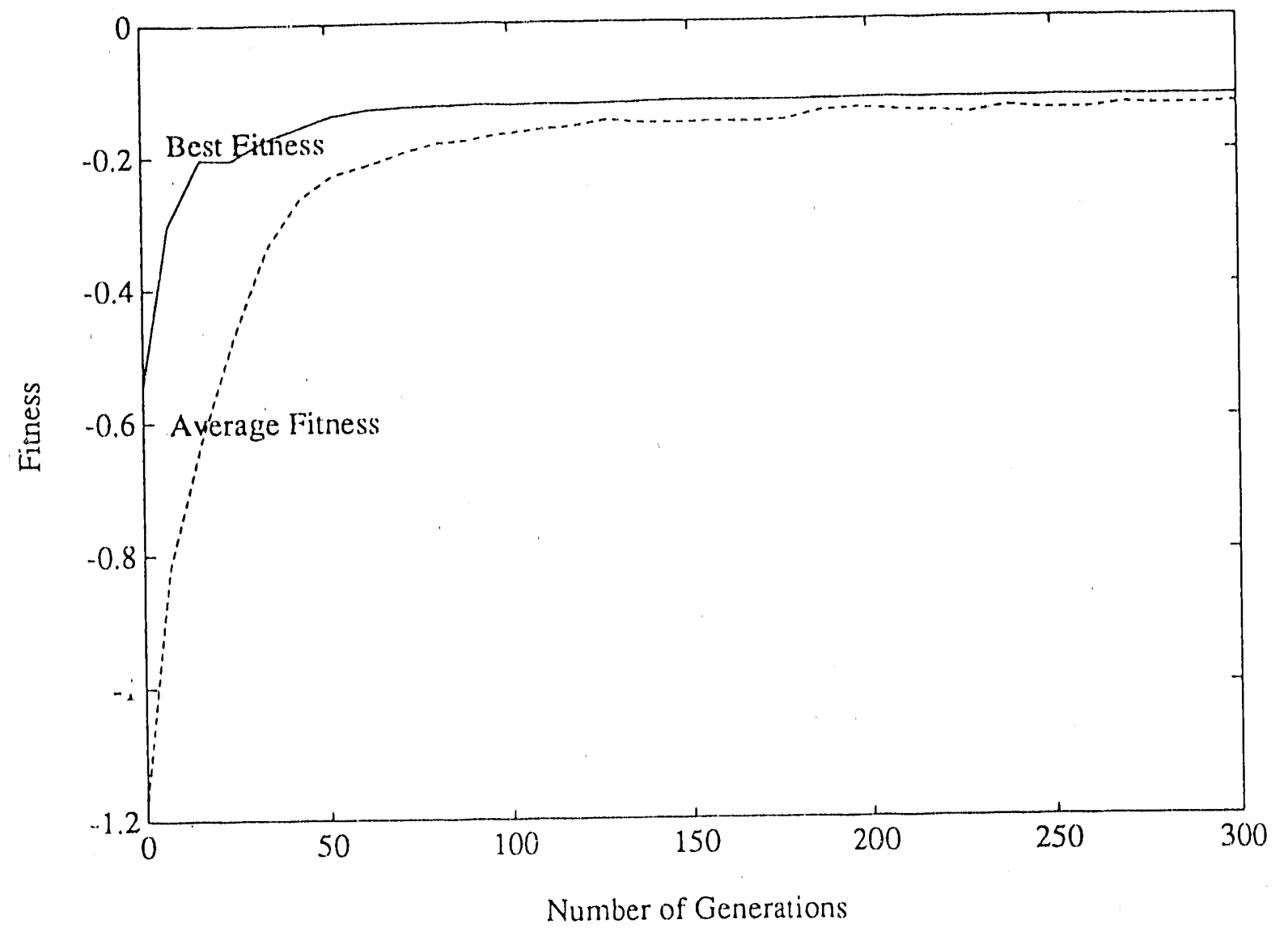




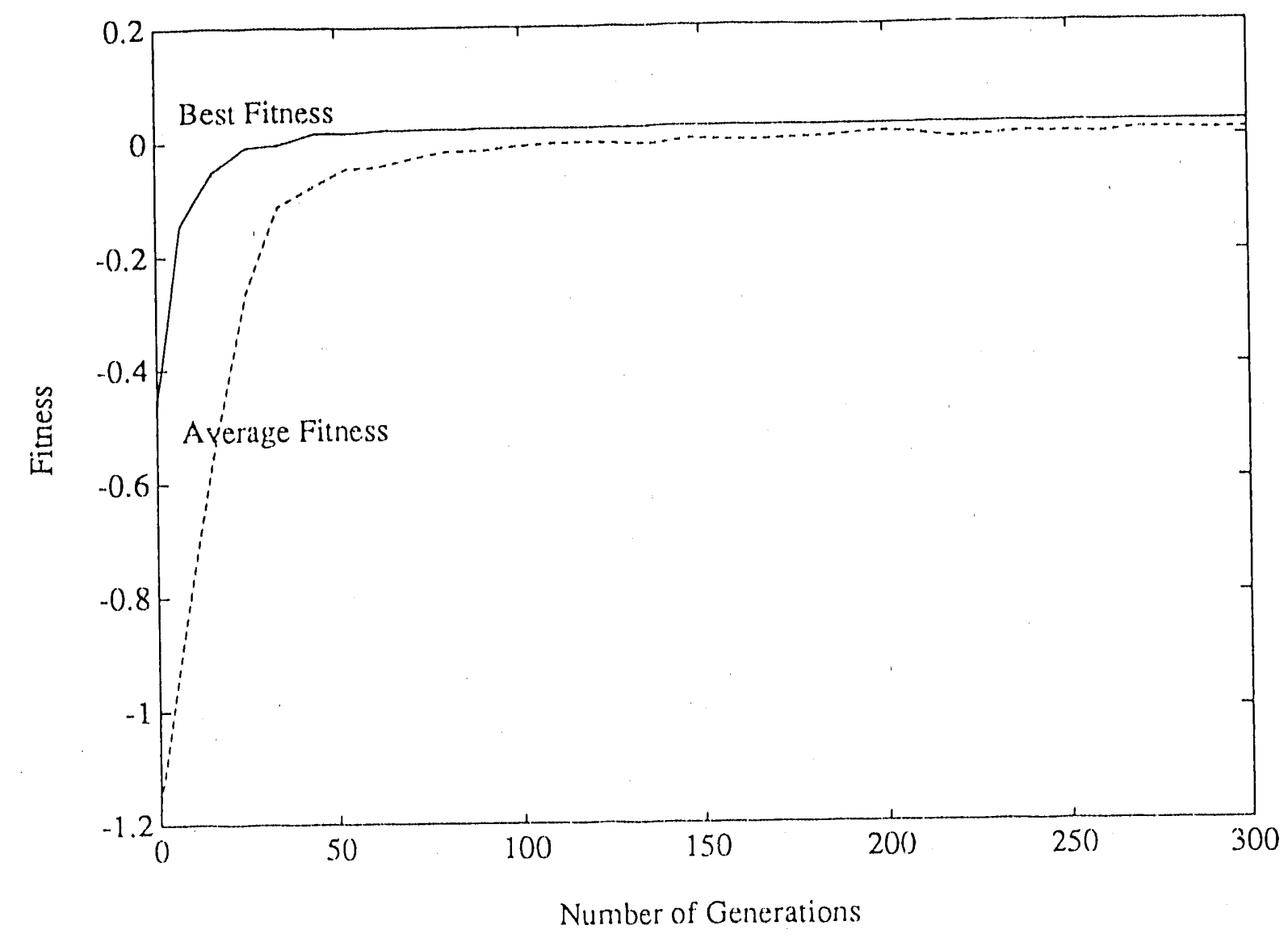



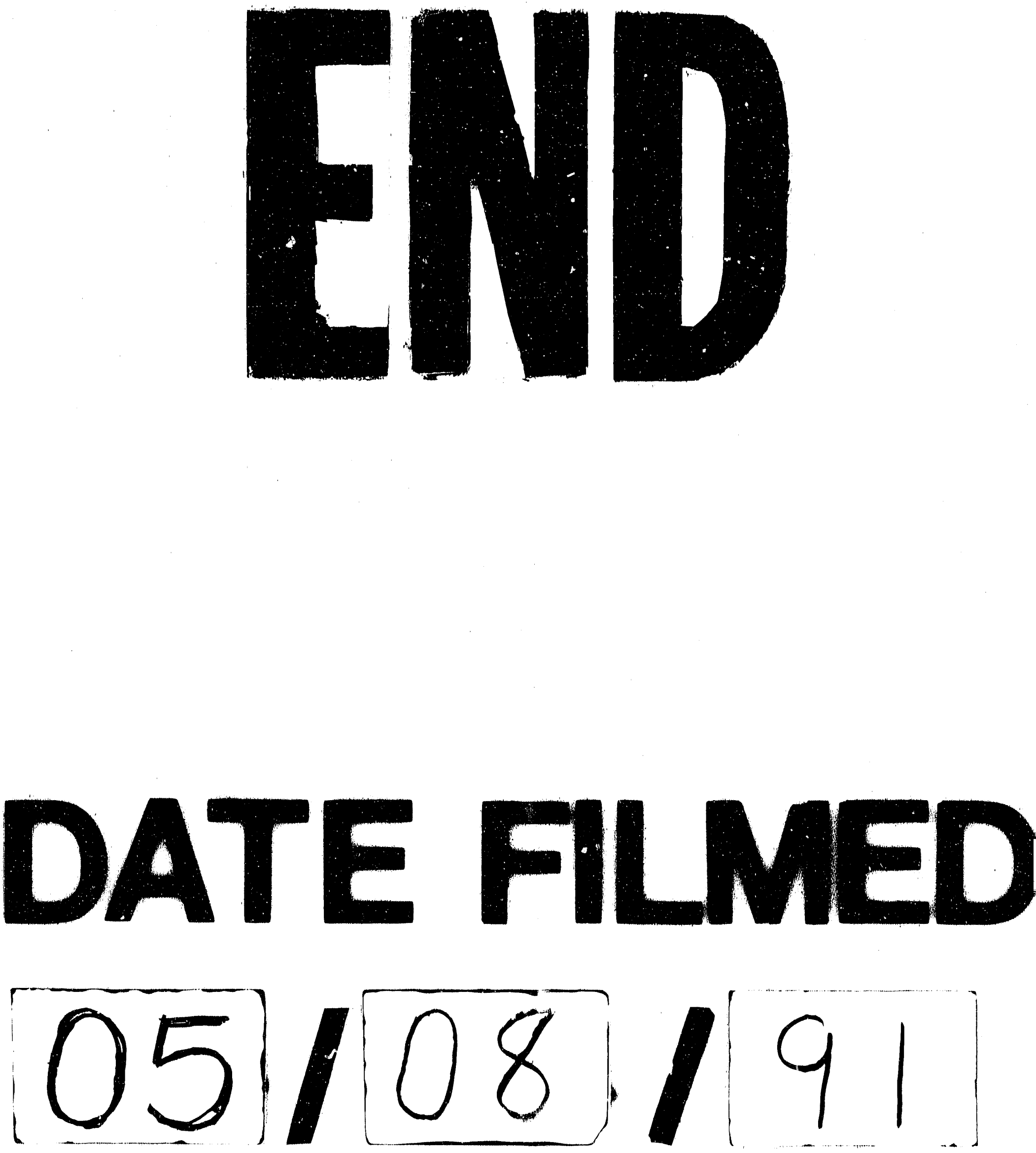
